\title{
Multiple Cranial Nerve Palsies, Without Definitive Brain Lesion. Rare Case of Plasma Cell Myeloma
}

${ }^{1}$ Uzma Nasim Siddiqui, ${ }^{2}$ Sadaf Andaleeb, ${ }^{3}$ Rabbia Abbass, Azeem Taj

Department of Medicine, Shaikh Zayed Medical Complex, Lahore

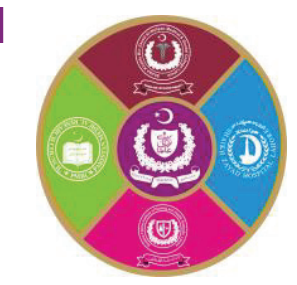

\begin{abstract}
Plasma cell myeloma rarely presents with neurological symptoms, and if this happens then cause is usually plasmacytoma. This is a very rare case to be presented as multiple cranial nerve palsies, unusual presentation of plasma cell myeloma without involvement of organic brain lesion in literature to date.

A 70 years old gentleman having ESRD, was received with diplopia, deviation of angle of mouth, on and off chest pain. Investigations ruled out stroke, cardiac event, autoimmune process or any other possible cause of cranial nerve palsies. Diagnosis of multiple myeloma was confirmed by immune electrophoresis which showed monoclonal gammopathy, punched out lytic lesions were found on skeletal scurvy and later confirmation was done by bone marrow biopsy for the presence of plasma cell myeloma.
\end{abstract}

ABSTRACT

Key words: plasma cell myeloma, cranial nerve palsies

\section{INTRODUCTION}

$\mathrm{P}$ lasma cell myeloma comprises $10 \%$ of hematological malignancies. ${ }^{1} \quad$ Common presentations of this disease are renal failure, anemia, bony lytic lesions, hyperviscosity and hypercalcemia. ${ }^{2}$ It rarely presents with neurological manifestation. $1 / 3$ of myeloma patients have clinical evidence of neuropathy at the time of presentation..$^{3,9}$ Cranial neuropathy is not a typical finding. Literature shows that these cranial nerve palsies are mostly secondary to soft tissue swellings like intra parenchymal leison ${ }^{4}$ or itself disease sequale leading to bony destruction. ${ }^{5}$ Brain involvement may be as a result of solitary cerebral plasmacytoma, or leptomeningial involvement. ${ }^{1}$ All these causes turned out to be negative in our patient therefore making this case unusual.

\section{CASE REPORT}

70 years old gentleman who was diagnosed as a case of end stage renal disease (ESRD) 6 months prior to the presentation, $\mathrm{He}$ was put on maintenance hemodialysis twice weekly, presented to us with deviation of mouth to left side and diplopia for the last 15 days. He was also suffering from generalized body aches and chest pain for 4 months, which usually relieved by taking pain killers. Decrease appetite and non documented weight loss was also one of his concern for the last few months. Examination showed a pale, ill looking gentleman with right sided upper motor neuron facial nerve palsy with failure of right eye abduction.

Initially diagnosis of stroke was made which turned out to be negative. We sent all base line investigations in which hypercalcemia was striking along with anemia and ESRD. Suspecting as a case of multiple myeloma, workup was initiated. X-ray skull showed punched out lytic lesions. Serum protein electrophoresis showed monoclonal gammapathyin the gamma region, further confirmation was done by bone marrow biopsy. Bone marrow revealed more than $70 \%$ infiltration by plasma cells with presence of binucleated and trinucleated forms. MRI brain did not show any new vascular insult, any meningeal enhancement, neither soft tissue swelling nor bony involvement was found. CSF analysis was done. MRI was also unremarkable even cytology did not show plasma cells. Autoimmune profile including ANA, ASMA, AMA, complement levels and RA factor were found to be negative. During hospital stay he was treated for hyperkalemia, hypercalcemia and anemia. Multiple hemodialysis sessions were done for uremia. Cardiac workup was done due to his chest pain. ECG, Echocardiography and cardiac enzymes were normal and did not show any evidence of ischemic heart disease.

After diagnosis of plasma cell myeloma was made, patient was referred to oncology department for initiation of chemotherapy and definitive management. 


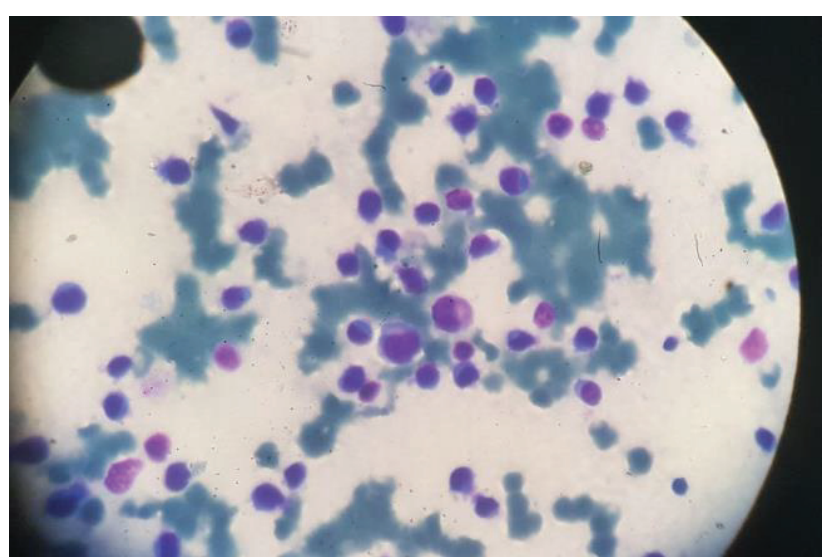

Fig-1: Bone marrow showing hypercellular marrow with abundant plasma cells, reduced erythropoiesis, myelopoesis and megakaryopoesis

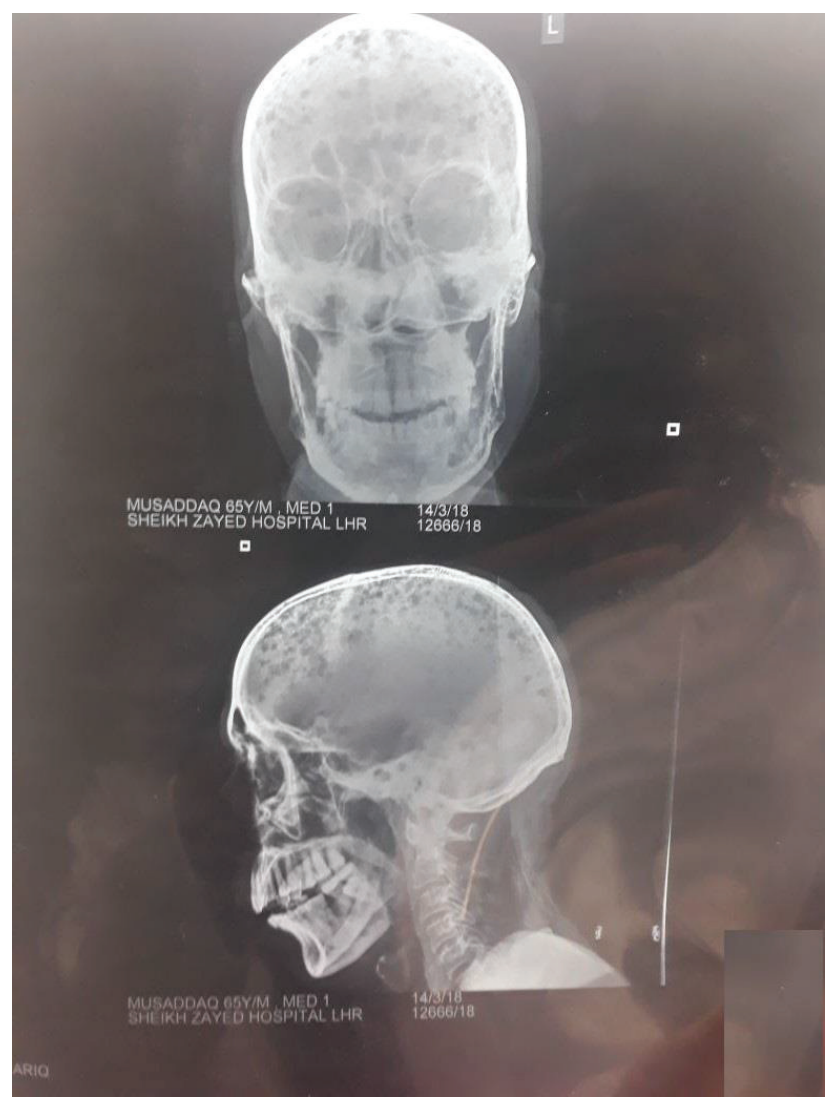

Fig-2: X ray skull AP and lateral view showing multiple punched out lytic lesions

\section{DISCUSSION}

Late diagnosis in multiple myeloma and untreated cases can lead to several complications and severe clinical manifestation like in our case where patient was having evidence of ESRD with body pain especially chest pain and but no diagnosis was made. Patient remained untreated and manifested as multiple cranial nerve palsies which is a late complication and rare manifestation of the underlying cause.
Diverse neurological findings may be associated in a patient with Multiple Myeloma, like metabolic encephalopathy as a result of uremia and hypercalcemia, Peripheral neuropathies and cranial nerve palsies. ${ }^{7}$ Cranial nerve palsies are uncommon and late complications of plasma cell myeloma, and if present they are manifestations of the presence of intracranial plasmacytoma. ${ }^{6}$ There are several cases in the literature with isolated cranial nerve palsies secondary to plasmacytoma, like petrous apex area leading to trigeminal and abducent nerve palsy ${ }^{4}$. Sphenoid sinus involvement leading to $6^{\text {th }}$ nerve palsy. ${ }^{7}$ Disease advancement can also result in bony destruction which can effect cranial nerve canal ${ }^{5}$, soft tissue swelling in right superior orbital fissure resulting in $3^{\text {rd }}$ nerve palsy, ${ }^{6}$ Abducent nerve palsy. ${ }^{2}$ This is unique case as index patient was having right sided $4^{\text {th }} 6^{\text {th }}$ and $7^{\text {th }}$ nerve palsies at time of presentation with no evidence of mass lesion, stroke, bony destruction, meningeal involvement. His MRI brain was non significant and all other possible causes of cranial nerve palsies were excluded, like cerebrovascular event, Vasculitis, or any other autoimmune etiology. CSF analysis was also done which was unremarkable with no plasma cell infiltration for possible leptomeningial myeloma. In literature several cases can be found with multiple cranial nerve palsies due to CNS involvement in various forms but in index case no neurological cause for cranial nerve palsies could be established, the only credible reason is possibility of nerve ischemia due to hyperviscosity in multiple myeloma. However this is an unusual presentation of multiple cranial nerve involvement without any substantial evidence of brain involvement. This is a rare patient from Pakistan being reported as multiple cranial nerves without definitive brain lesion or leptomeningeal involvement.

\section{REFERENCES}

1. Chakrabarti S, Pan K, Panchadhyayee S, Halder S, Chowdhury R. Multiple myeloma presenting as isolated sixth nerve palsy. Int J Res Med Sci. 2014; 2(2):738-40.

2. Singh TS, Gurudath GS, Singh TB, Singh NB. Multiple cranial nerve palsies: As first presentation of multiple myeloma with intracranial plasmacytoma.Jams.2012;2(4):129-31

3. Sahoo LK, Biswal NR, Mallik AK, Mohanty G, Swain KP. Bilateral sixth cranial nerve palsy as a presenting feature of multiple myloma: A rare presentation. J Appl Hematol. 2015; 6:130-2.

4. Thiruvengadam SS, Prayson RA. Multiple myeloma presenting with unilateral abducens and 
trigeminal nerve palsies. Journal of Clinical Neuroscience. 2016 Apr 1; 26:143-4.

5. Newman NB, Puthenpura V, Mischell S, Ferreira G. Hypoglossal Nerve Mononeuropathy as the First Presenting Symptom of Progressing Multiple Myeloma. World journal of oncology. $2017 \mathrm{Feb} ; 8(1): 15$.

6. León-Ruiz M, Benito-León J, Sierra-Hidalgo F, García-Soldevilla MÁ, Izquierdo-Esteban L, Tejeiro-Martinez J, Cabrera-Valdivia F, GarcíaAlbea ER. First case described of isolated, complete and fluctuating cranial nerve III palsy heralding multiple myeloma. Revista de neurologia. 2015 Feb; 60(3):115-9.

7. Pak Neda et al. Mutiple cranial nerve palsy concomitant with leptomeningeal involvement in multiple myeloma: A case report and review of literature. IJHOSCR. 2018; (12)1:8-13.

8. Bellan LD, Cox TA, Gascoyne RD. Parasellar syndrome caused by plasma cell leukemia. Canadian Journal of Ophthalmology. Journal Canadiend'Ophtalmologie.1989 Dec;24(7):331-4.

9. Abducens Nerve palsy as initial Presentation of Multiple Myeloma and Intracranial Plasmacytoma. J Clin Med. 2018 Sep; 7(9):253.
The Authors:

Dr. Uzma Nasim Siddiqui

Assistant Professor,

Department of Medicine,

Shaikh Zayed Medical Complex, Lahore.

Dr. Sadaf Andaleeb

Fellow Rheumotology,

Shaikh Zayed Medical Complex, Lahore.

Dr. Rabbia Abbass

Trainee Registrar,

Department of Medicine,

Shaikh Zayed Medical Complex, Lahore.

Prof. Azeem Taj

Head, Department of Medicine,

Shaikh Zayed Medical Complex, Lahore.

Corresponding Authors:

Dr. Uzma Nasim Siddiqui

Assistant Professor,

Department of Medicine,

Shaikh Zayed Medical Complex, Lahore.

Email: Uzmamamoon@gmail.com

ADDENDUM
Proceedings S.Z.P.G.M.I. Vol: 33(3): pp. 15-19, 2019. PSZMC-716-33-3-2019
Seroprevalence of Varicella Zoster Virus Immunoglobulin G Among Asymptomatic
Healthcare workers
Authors: Saira Zafar, Muhammad Imran, Sadia Haleema, Hirra Ghaffar, Namra Yunus, Mariya
Farooq have hereby added the omitted "Acknowledgement" to the print version of the
manuscript.
This research was carried out at the Department of Microbiology, Shaikh Zayed Hospital,
Lahore. The authors are highly thankful to the entire staff of Microbiology Department who
helped in this regard.

